\section{Microambiente medular en la leucemia mieloide crónica: su relación con la enfermedad y la respuesta al tratamiento}

JOSÉ ALEJANDRO ARISTIZÁBAL 1,2,a , MAURICIO CHANDIA ${ }^{1,2,3, b}$, MARÍA CONSUELO DEL CAÑIZO ${ }^{1,2, \mathrm{c}}$, FERMÍN SÁNCHEZ-GUIJO ${ }^{1,2, \mathrm{c}}$

\section{Bone marrow microenvironment in chronic myeloid leukemia: Implications for disease physiopathology and response to treatment}

Chronic myeloid leukemia (CML) is a myeloproliferative neoplasm related to the presence of the BCR-ABL1 fusion gene, linked to $t$ (9;22) (q34;q11). It is originated from an abnormal hematopoietic stem cell, which is characterized as its normal counterparts by long-term self-renewal and multi-lineage differentiation. Both leukemic and quiescent normal hematopoietic stem cells preferentially reside in the osteoblastic niche. Mesenchymal stromal cells (MSC) are located near them, playing a critical role in their regulation. Currently, with tyrosine kinase inhibitor (TKI) therapy, long term clinical responses are achieved in most CML cases. However, late treatment failures may be observed related to the persistence of leukemic stem cells. The interactions between the leukemic stem cell and the microenvironment may be responsible in part for these events. We review the interactions between the leukemic stem cell and BM stroma and its potential clinical and therapeutic implications.

(Rev Med Chile 2014; 142: 599-605)

Key words: Drug resistance, neoplasm; Leukemia, myeloid; Mesenchymal stromal cells; Stem cells; Stem cell niche.
'Servicio de Hematología, Hospital Universitario de Salamanca, Salamanca, España. ${ }^{2}$ Instituto de Investigaciones Biomédicas de Salamanca (IBSAL), Salamanca, España. ${ }^{3}$ Becario Fundación BBVAFundación Carolina.

aBacteriólogo, Máster en Biología y Clínica del Cáncer, Universidad de Salamanca.

bMédico hematólogo, Máster en Biología y Clínica del Cáncer, Universidad de Salamanca. 'Médico hematólogo, PhD, Universidad de Salamanca.

Recibido el 19 de julio de 2013, aceptado el 29 de noviembre de 2013.

Correspondencia a:

Dr. Fermín Sánchez-Guijo Servicio de Hematología, IBSAL-Hospital Universitario de Salamanca, Paseo de San Vicente 58-182, 37007 Salamanca, España.

ferminsg@usal.es
L a leucemia mieloide crónica (LMC) es una neoplasia mieloproliferativa cuyo origen está relacionado con la adquisición del gen de fusión $B C R-A B L 1$, asociado a la $\mathrm{t}(9 ; 22)$ $(\mathrm{q} 34 ; \mathrm{q} 11)^{1}$. Representa cerca de $15-20 \%$ de los casos de las leucemias en el adulto, aparece frecuentemente hacia quinta o sexta década de la vida y su incidencia es de 1-2 casos por 100.000 habitantes por año ${ }^{2,3}$. La enfermedad sigue un curso progresivo, que se inicia con la fase crónica que es indolente, seguida de una trasformación de la enfermedad hacia la denominada fase acelerada y finalmente la crisis blástica, a la que se llega en todos los casos de no mediar una terapia efectiva.

El tratamiento actual con inhibidores de ti- rosina quinasa (ITK) como el imatinib mesilato (IM) es capaz de lograr una supervivencia libre de progresión de $81 \%$ a los 8 años de acuerdo con los datos del estudio IRIS 4 . El IM ha demostrado ser útil incluso en adultos mayores con comorbilidades asociadas, logrando respuestas similares a los pacientes jóvenes en estudios observacionales ${ }^{5}$. Sin embargo, debe considerarse que del $45 \%$ de los pacientes que abandonaron el estudio IRIS en el período de seguimiento señalado, un tercio lo hizo por "resultados clínicos no satisfactorios", lo que sugiere que aún existe un grupo de pacientes en los cuales el mecanismo de acción del fármaco no ha logrado el objetivo terapéutico.

La resistencia a la acción de los ITK puede 
originarse en factores intrínsecos y extrínsecos. Los factores intrínsecos son aquellos relacionados con características propias de las células tumorales, tales como mutaciones puntuales en el dominio tirosina quinasa del gen $B C R-A B L 1$, sobreexpresión de este gen de fusión, evolución clonal y la persistencia de células stem leucémicas (CSL) ${ }^{6}$. Los factores extrínsecos son aquellos que no dependen de la acción directa del $B C R-A B L 1$ y pueden deberse a menor biodisponibilidad de la droga y efectos favorecedores del micromedioambiente sobre el clon tumoral ${ }^{7}$.

En esta revisión describiremos las características principales de la CSL y cómo su relación con el micromedioambiente medular y las células estromales mesenquimales (MSC) pueden afectar su respuesta al tratamiento.

\section{Fisiopatología del $B C R / A B L$ en el desarrollo de la LMC}

La oncoproteína de fusión $B C R / A B L$ se distribuye a través de todo el citoplasma e interactúa con varias vías de señalización relacionadas con funciones de proliferación, diferenciación y supervivencia. Una de la proteínas con las que interacciona es Ras, la cual se activa con la ayuda de varias proteínas mediadoras. Una vez que se auto-fosforila la proteína de fusión en su tirosina 177, se genera el sitio de unión del dominio SH2 (SRC Homology-2) que interactúa con la proteína adaptadora GRB2 (growth factor receptor-boundprotein 2). A su vez, la proteína GRB2 interactúa con la proteína SOS (son of sevenless). El complejo BCR-ABL-GRB2-SOS resultante se asocia con Ras y estimula su activación. Las proteínas adaptadoras CRKL (CRK-like) y SHC (SH2-containing protein) pueden también mediar la activación del Ras a través de su interacción con el $B C R-A B L 1^{8}$.

Una vez activado Ras a través de la vía de MAPK (mitogen activated protein kinase) se asocia con Raf, el cual cataliza la fosforilación de MEK1 y MEK2 (mitogen-activated and extracellularsignal regulated kinases 1 and 2) lo que estimula la expansión del clon tumoral independientemente de la acción de factores de crecimiento. Otra vía estimulada por el $B C R-A B L 1$ es la del PI3K, lo que lleva a la supresión de la muerte celular programada. $B C R-A B L 1$ también se asocia con componentes de adhesión focal como las FAK (focal adhesion kinases) a través del complejo CRK-FAK-PYK2, lo que favorece la disminución de la adhesión celular. Otras acciones descritas para el BCR$A B L 1$ son la activación de la vía del JAK-STAT y la interacción con proteínas antiapoptóticas de la familia del $B C L-2^{8}$.

\section{Célula stem de la LMC}

La existencia de células stem hematopoyéticas de médula ósea (MO) se demostró a inicios de los años sesenta, cuando McCulloch y Till observaron que al inyectar células de $\mathrm{MO}$ en ratones irradiados se formaban nódulos esplénicos que posteriormente generaban hematopoyesis ${ }^{9}$. Dick y cols demostraron posteriormente la existencia de una célula stem leucémica tras reproducir una leucemia mieloide aguda al trasplantar células $\mathrm{CD} 34^{+} \mathrm{CD} 38^{-}$en ratones inmunocomprometidos $\mathrm{NOD} / \mathrm{SCID}^{10}$. Por medio de técnicas citogenéticas y moleculares se ha demostrado el origen de la LMC en una célula stem al demostrar la presencia del gen de fusión en poblaciones de granulocitos, monocitos, precursores eritroides, megacariocitos y linfocitos ${ }^{11,12}$. Otros autores han encontrado mRNA de $B C R-A B L 1$ en subpoblaciones de precursores CD34+ de pacientes con LMC, lo que sugiere que la CSL sería muy parecida a la célula stem hematopoyética normal de la médula ósea $(\mathrm{CSH})$, pero con la translocación específica ${ }^{6,13}$.

Algunas características intrínsecas de la CSL de pacientes con LMC pueden explicar su resistencia al tratamiento. Así, se ha comprobado que expresan niveles menores de OCT-1 (organic cation transporter-1), que es un transportador involucrado en la captación activa del IM al interior de la célula ${ }^{14,15}$. A pesar de ello, la menor actividad de OCT-1 en las CSL no ha podido correlacionarse con una menor probabilidad de respuesta molecular mayor a los 12 meses $^{16}$. Otro de los mecanismos implicados en el mantenimiento de la autorrenovación de la célula stem es la vía $\mathrm{Wnt} /$ beta-catenina, producto de la acción de $B C R-A B L 1^{17}$. Se ha demostrado en modelos murinos de xeno-trasplantes que la abolición de esta vía previene el desarrollo de la LMC a partir de la CSL. Abe y cols, al analizar las células de MO purificadas de pacientes con LMC en tratamiento, observaron que los transcriptos del oncogén de fusión persistían únicamente en los progenitores 
hematopoyéticos, mientras desaparecían en los más diferenciados, lo que sugiere una mayor resistencia al tratamiento con ITK en las $\mathrm{CSL}^{18}$. Por otro lado, se ha demostrado que sobrenadantes de medio de cultivo de líneas celulares resistentes a la acción de imatinib puede proteger a las células tumorales de pacientes con LMC vírgenes a tratamiento de la muerte celular inducida por imatinib o nilotinib; mecanismo que podría estar mediado por un aumento de la secreción autocrina de GM-CSF y podría contribuir a la resistencia al tratamiento ${ }^{19}$.

En la crisis blástica pueden aparecer poblaciones tumorales de diferentes linajes hematopoyéti$\cos$ (linfoides B, linfoides $\mathrm{T}$, mieloides o de linaje ambiguo) que obedecerían a una evolución clonal diferente. En esta etapa, células de progenie más diferenciada como el GMP (progenitor granulomonocítico), adquiere características de célula "stem" como la auto-renovación, por medio de la activación de la vía del Wnt/ $\beta$-catenina, lo que las hacen intrínsecamente más resistentes a los ITK ${ }^{20}$.

\section{Micromedioambiente medular}

El estroma medular tiene como función principal la producción de la matriz extracelular, citoquinas y factores de crecimiento que ejercen una función regulatoria sobre las células hematopoyéticas. Las CSH interactúan a través de receptores específicos de superficie celular tanto con la matriz extracelular, como con moléculas de superficie de las células del nicho, lo que refleja la estrecha relación entre ambos componentes celulares de la $\mathrm{MO}^{21}$.

En condiciones normales la CSH reside cerca del endostio, protegida por los osteoblastos (nicho

Tabla 1. Características del microambiente medular de la LMC que influyen en la respuesta al tratamiento con ITK en la LMC

- Disminución de la capacidad de soporte de la hematopoyesis normal

- Protección frente la apoptosis

- Mantenimiento de la quiescencia de las células stem tumorales

- Inducción de resistencia mediada por citoquinas e interacciones célula-célula osteoblástico), mientras que los progenitores más diferenciados se sitúan cerca de los sinusoides (nicho vascular). El nicho osteoblástico se ubica principalmente en las trabéculas óseas y provee a la CSH del micromedioambiente necesario para su quiescencia. A su vez, el nicho vascular propicia la diferenciación y la proliferación ${ }^{21,22}$. Ambos nichos están muy relacionados anatómica y funcionalmente, por lo que muchos autores consideran que serían una unidad funcional ${ }^{23}$.

Una vez en el nicho osteoblástico, la CSHse mantiene allí gracias a la expresión de moléculas de adhesión. Especialmente importantes son la interacción entre el CXCR4 (expresado en la CSH) y el CXCL12 (que se expresa en las MSC y los osteoblastos) y la VLA-4 de la CSH, que interactúa con la fibronectina de la matriz extracelular ${ }^{24,25}$. A nivel del osteoblasto $\mathrm{N}$-cadherina (+), la CSH interactúa a través de las proteínas Beta-catenina/ $\mathrm{N}$ cadherina y la Tie2/Angiopoyetina 1, las que experimentalmente al ser suprimidas favorecen la maduración de la $\mathrm{CSH}^{23}$. En el nicho vascular las $\mathrm{CSH}$ carecen de N-cadherina, por lo que se mantienen unidas a las células que expresan CXCL12 mediante la interacción CXCR4-CXCL12. Así, se evidencia que tanto a nivel osteoblástico como a nivel vascular la acción del CXCL-12 (SDF-1) es fundamental para el mantenimiento de las $\mathrm{CSH}^{24}$ (Figura 1).

\section{Características del microambiente de la LMC y su relación con las CSL}

La CSL de la LMC y de la LMA anidan en el nicho hematopoyético mediante moléculas de adhesión como CD44 y VLA-4 y utilizan las mismas vías que las $\mathrm{CSH}$ normales para su supervivencia ${ }^{26}$. Un hallazgo reciente sugiere que factores propios del micromedioambiente medular de la LMC pueden aumentar la expresión de la vía de del Wnt/ $\beta$ catenina por medio de la unión de $\mathrm{N}$-cadherinas a las MSC, lo que confiere a las CSL protección contra la acción de los ITK ${ }^{27}$.

Además, el estroma medular de la LMC tiene varias características que pueden favorecer la supervivencia y crecimiento del clon tumoral. Se ha demostrado que la capacidad de proliferación de las CSH normales estaría disminuida en contacto con el estroma de LMC, a diferencia de lo que ocurre con las CSL, en las que se mantiene inalterada ${ }^{28}$. 


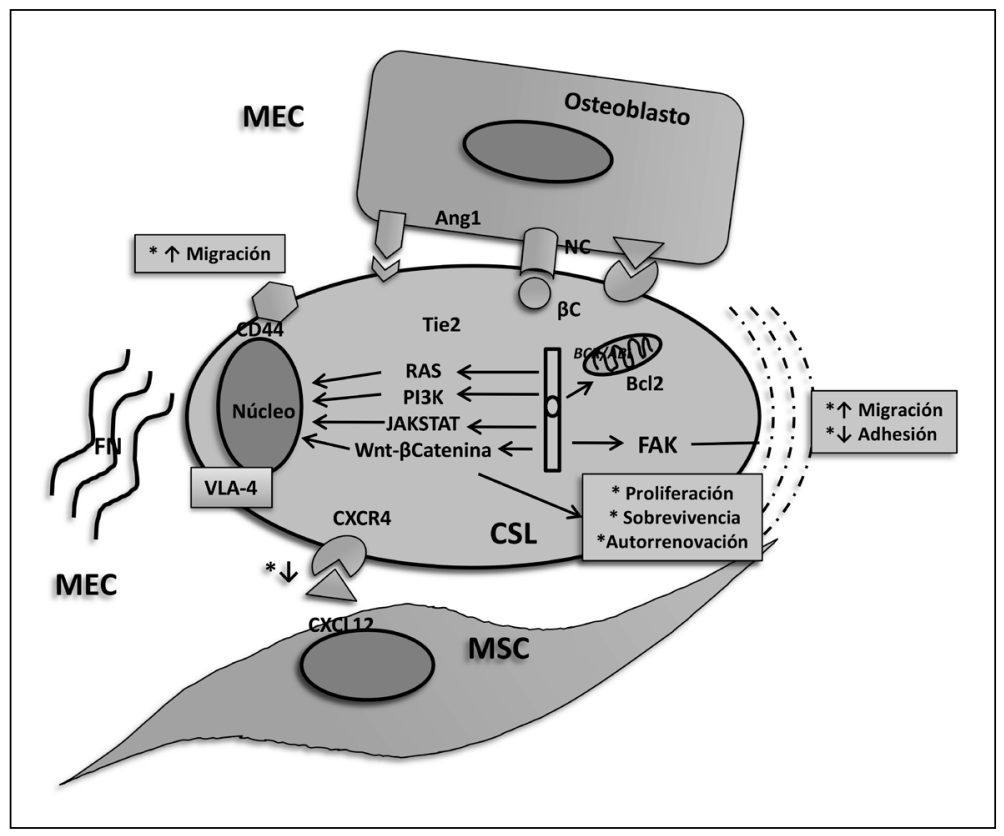

Figura 1. Efectos directos del $B C R /$ $A B L$ sobre la célula stem leucémica e interacciones de ésta con el nicho hematopoyético: $B C R / A B L$ activa una serie de vías que estimulan la supervivencia y proliferación del clon tumoral y, por otro lado, disminuyen la adhesión, favoreciendo la migración de la célula. La CSL se mantiene en estrecha relación con su medio a través de moléculas de adhesión que interactúan con moléculas de la matriz extracelular y receptores de superficie de las células estromales mesenquimales y los osteoblastos. CSL: Célula Stem Leucémica; MSC: Célula estromal Mesenquimal; MEC: matriz extracelular; FAK: Focal adhesion Kinases; Ang1: Angiopoyetina 1; FN: Fibronectina; NC: N-Cadherina; $\beta$-Catenina. *denota efectos inducidos por el $B C R /$ $A B L$ sobre la célula tumoral. Las líneas de punto y raya denotan alteraciones en la función de las FAK.

\section{Células mesenquimales estromales (MSC) y LMC}

Las MSC constituyen el $0,01-0,001 \%$ de las células nucleadas de la $\mathrm{MO}^{29}$. De acuerdo a la definición propuesta por la Sociedad Internacional de Terapia Celular, las MSC para ser consideradas como tales deben cumplir con los requisitos de poseer adherencia al plástico en cultivos estándar, tener un inmunofenotipo positivo para marcadores estromales (CD73, CD105, CD90) en ausencia de antígenos hematopoyéticos (CD34, CD45, CD14, CD19 y HLA-DR) y capacidad demostrable de diferenciación a otros tipos celulares como osteoblastos, adipocitos y condrocitos ${ }^{30}$.

Una de las principales funciones de las MSC en la MO normal es la creación de un microambiente propicio para la hematopoyesis. En este sentido, hemos observado en nuestro grupo que las MSC de donantes sanos pueden restaurar la función del estroma en modelos in vivo de $\mathrm{MO}$ dañada por etopósido ${ }^{31}$. Las MSC también secretan citoquinas y factores de crecimiento, como el M-CSF, el ligando del FLT-3, el stem cell factor (SCF) e interleuquinas como IL-6, IL-7, IL-11, IL-12, IL-14 y IL-15 $5^{32}$.

A pesar de las diferencias que se han observado entre las MSC de otras hemopatías como los síndromes mielodisplásicos ${ }^{33}$, mieloma múltiple y las MSC normales ${ }^{34}$, los datos existentes sobre las MSC de LMC son controvertidos. Zhao et al han observado que las MSC de LMC son similares a MSC normales en el fenotipo, la morfología y capacidad de diferenciación multilineal ${ }^{35}$. En lo que coinciden la mayoría de los autores es que las MSC de LMC, al menos aquellas caracterizadas in vitro siguiendo los criterios de la Sociedad internacional de Terapia Celular que hemos indicado, no poseen el gen de fusión $B C R-A B L 1^{36}$. Sin embargo, otros autores afirman que las MSC de LMC no son completamente similares a las células de donantes sanos, lo que concuerda con datos aún no publicados por nuestro grupo ${ }^{37,38}$.

En el nicho medular las células tumorales mantienen su potencial proliferativo y están protegidas de la apoptosis ${ }^{28}$. La migración de las células tumorales de la LMC a este nicho se logra por medio de la interacción con las MSC a través del eje CXCR4-CXCL12 ${ }^{39}$. Se ha observado en mieloma múltiple y en otras hemopatías que las MSC promueven la angiogénesis y tienen un perfil de expresión génica diferente a las normales, acción aún no documentada en LMC, pero que podrían estar implicada en su patogenia ${ }^{40,41}$.

Las MSC normales han mostrado efectos inhibitorios en la activación y proliferación de 
linfocitos T por medio de la inducción de bloqueos en fases G0/G1. Las MSC de pacientes con LMC comparadas con MSC normales mostraron en ensayos in vitro menor capacidad de inhibición de expresión de marcadores de activación de linfocitos T. Esto puede indicar que las MSC de LMC tienen menor capacidad inmunomoduladora, lo que apoyaría de nuevo su alteración funcional ${ }^{42}$.

Asimismo se ha observado que la función tirosina quinasa del $B C R-A B L 1$ tiene efectos en la disminución de la adhesión celular y aumento de la migración ${ }^{8}$ Este efecto estaría mediado por una baja expresión de CXCR4 en las células de LMC que pueden interferir en la interacción con el CXCL12 producido por las $\mathrm{MSC}^{43}$. El tratamiento con imatinib tiene la capacidad de restaurar la expresión de CXCR4 en las células tumorales, lo que les hace más susceptibles de migrar al nicho y desarrollar resistencia a la acción del medicamento, la que puede ser revertida con el uso de antagonistas del CXCR4 y de integrinas ${ }^{43}$. El inhibidor de CXCR4 plerixafor induce la migración y reduce la adhesión de las células tumorales de la LMC a componentes de la matriz extracelular y células estromales de medula ósea. En estudios in vitro con este fármaco, Weisberg et al observaron que el plerixafor disminuye la resistencia a nilotinib de células de LMC en co-cultivo con células estromales de $\mathrm{MO}^{44}$. Estos resultados apoyan la idea de que el uso de agentes que bloquean la interacción nicho-célula hematopoyética en conjunto con ITK podría disminuir la resistencia al tratamiento y favorecer la erradicación de la enfermedad mínima residual.

De acuerdo a lo anteriormente expuesto, las diversas interacciones entre la CSL y la MSC pueden influenciar la respuesta al tratamiento con ITK, y así favorecer una respuesta inadecuada inicial o las recaídas tardías de la enfermedad (Tabla 1) ${ }^{44}$.

\section{Conclusiones}

Con la aparición de los ITK, el tratamiento de la LMC ha experimentado un cambio radical. Sin embargo, persisten factores no completamente identificados que son potencialmente responsables de la falta de respuesta y progresión de la enfermedad. La persistencia de CSL resistentes a la acción de los ITK y el efecto favorecedor que el estroma medular y las MSC ejerce sobre ellas pueden ex- plicar en parte estas resistencias. Por este motivo los estudios defuturas terapias en desarrollo para LMC con intención curativa deberían considerar tanto al micromedioambiente como a las CSL posibles dianas terapéuticas.

\section{Referencias}

1. Swerdlow SH, Campo E, Harris NL, Jaffe ES, Pileri SA, Stein $\mathrm{H}$, et al. World Health Organization Classification of Tumours of Haematopoietic and Lymphoid Tissues, IARC Press, Lyon, 2008; p 32-7.

2. Siegel R, Naishadham D, Jemal A. Cancer statistics, 2012. CA Cancer J Clin 2012; 62: 10-29.

3. Sant M, Allemani C, Tereanu C, De Angelis R, Capocaccia R, Visser O, et al. HAEMACARE Working Group. Incidence of hematologic malignancies in Europe by morphologic subtype: results of the HAEMACARE project. Blood 2010; 116: 3724-34.

4. Kantarjian H, Sawyers C, Hochhaus A, Guilhot F, Schiffer C, Gambacorti-Passerini C, et al. Hematologic and Cytogenetic Responses to Imatinib Mesylate in Chronic Myelogenous Leukemia. N Engl J Med 2002; 346: 64552.

5. Sánchez-Guijo FM, Durán S, Galende J, Boqué C, Nieto JB, Balanzat J, et al. Evaluation of tolerability and efficacy of imatinib mesylate in elderly patients with chronic phase CML: ELDERGLI study. Leuk Res 2011; 35: 11847.

6. Stuart S, Minami Y, Wang J. The CML stem cell. Evolution of the progenitor. Cell Cycle 2009; 8: 1338-43.

7. Apperley JF. Part I: mechanisms of resistance to imatinib in chronic myeloid leukaemia. Lancet Oncol 2007; 8: 1018-29.

8. Weisberg E, Manle PW, Cowan-Jacob SW, Hochhaus A, Griffin JD. Second generation inhibitors of BCR-ABL for the treatment of imatinib-resistant chronic myeloid leukaemia. Nature Rev Cancer 2007; 7: 345-56.

9. Till JE, McCulloch EA. A direct measurement of the radiation sensitivity of normal mouse bone marrow cells. Radiat Res 1961; 14: 213-22.

10. Bonnet D, Dick JE. Human acute myeloid leukemia is organized as a hierarchy that originates from a primitive hematopoietic cell. Nat Med 1997; 3: 730-7.

11. Primo D, Sánchez ML, Espinosa AB, Tabernero MD, Rasillo A, Sayagués JM, et al. Lineage involvement in chronic myeloid leukaemia: comparison between MBCR/ $A B L+$ and $\mathrm{m} B C R / A B L+$ cases. Br J Haematol 2006; 132: 736-9.

12. Haferlach T, Winkemann M, Nickenig C, Meeder M, 
Ramm-Petersen L, Schoch R, et al. Which compartments are involved in Philadelphia-chromosome positive chronic myeloid leukaemia? An answer at the single cell level by combining May-Grünwald-Giemsa staining and fluorescence in situ hybridization techniques. Br J Haematol 1997; 97: 99-106.

13. Maguer-Satta V PA, Eaves AC, Eaves CJ. BCR-ABL expression in different subpopulations of functionally characterized $\mathrm{Ph}+\mathrm{CD} 34+$ cells from patients with chronic myeloid leukemia. Blood 1996; 88: 1796-804.

14. Brendel C, Scharenberg C, Dohse M, Robey R, Bates $S$, Shukla $S$, et al. Imatinib mesylate and nilotinib (AMN107) exhibit high-affinity interaction with ABCG2 on primitive hematopoietic stem cells. Leukemia 2007; 21: 1267-75.

15. Jiang X, Zhao Y, Smith C, Gasparetto M, Turhan A, Eaves A, et al. Chronic myeloid leukemia stem cells possess multiple unique features of resistance to $B C R-A B L$ targeted therapies. Leukemia 2007; 21: 926-35.

16. Engler JR, Frede A, Saunders VA, Zannettino ACW, White DL, Hughes TP. The poor response to imatinib observed in CML patients with low OCT-1 activity is not attributable to lower uptake of imatinib into their CD34 (+) cells. Blood 2010; 116: 2776-8.

17. Zhao C, Blum J, Chen A, Kwon HY, Jung SH, Cook JM, et al. Loss of beta-catenin impairs the renewal of normal and CML stem cells in vivo. Cancer Cell 2007; 12: 528-41.

18. Abe A, Minami Y, Hayakawa F, Kitamura K, Nomura $\mathrm{Y}$, Murata $\mathrm{M}$, et al.Retention but significant reduction of BCR-ABL transcript in hematopoietic stem cells in chronic myelogenous leukemia after imatinib therapy. Int J Hematol 2008; 88: 471-5.

19. Wang Y, Cai D, Brendel C, Barett C, Erben P, Manley $\mathrm{PW}$, et al. Adaptive secretion of granulocyte-macrophage colony-stimulating factor (GM-CSF) mediates imatinib and nilotinib resistance in $B C R / A B L+$ progenitors via JAK-2/STAT-5 pathway activation. Blood 2007; 109: 2147-55.

20. Minami Y, Stuart SA, Ikawa T, Jiang Y, Banno A, Hunton IC, et al. BCR-ABL ransformed GMP as myeloid leukemic stem cells. Proc Natl Acad Sci USA 2008; 105: 17967-72.

21. Calvi LM, Adams GB, Weibrecht KW, Weber JM, Olson DP, Knight MC, et al. Osteoblastic cells regulate the haematopoietic stem cell niche. Nature 2003; 425: 841-6.

22. Nilsson SK, Johnston HM, Coverdale JA. Spatial localization of transplanted hemopoietic stem cells: inferences for the localization of stem cell niches. Blood 2001; 97: 2293-9.

23. Wang H, Zhang P, Liu L, Zoul L. Hierarchical organization and regulation of the hematopoietic stem cell osteoblastic niche. Crit Rev Oncol Hematol 2013; 85: $1-8$.

24. Nair RR, Tolentino J, Hazlehurst LA. The bone marrow microenvironment as a sanctuary for minimal residual disease in CML.Biochem Pharmacol 2010, 80; 602-12.

25. Matsunaga T, Takemoto N, Sato T, Takimoto R, Tanaka I, Fujimi A, et al. Interaction between leukemic-cell VLA-4 and stromal fibronectin is a decisive factor for minimal residual disease of acute myelogenous leukemia. Nat Med 2003; 9: 1158-65.

26. Krause DS, Lazarides K, von Andrian UH, Van Etten RA. Requirement for CD44 in homing and engraftment of BCR-ABL-expressing leukemic stem cells. Nat Med 2006; 12: 1175-80.

27. Zhang B, Li M, McDonald T, Holyoake TL, Moon RT, Campana D, et al. Microenvironmental protection of CML stem and progenitor cells from tyrosine kinase inhibitors through $\mathrm{N}$-Cadherin and Wnt-beta-catenin signaling. Blood 2013; 121: 1824-38.

28. Bhatia R, McGlave PB, Dewald GW, Blazar BR, Verfaillie $\mathrm{CM}$. Abnormal function of the bone marrow microenvironment in chronic myelogenous leukemia: role of malignant stromal macrophages. Blood 1995; 85: 363645.

29. Dazzi F, Ramasamy R, Glennie S, Jones SP, Roberts I. The role of mesenchymal stem cells in haemopoiesis. Blood Rev 2006; 20: 161-71.

30. Dominici M, Le Blanc K, Mueller I, Slaper-Cortenbach I, Marini F, Krause D, et al. Minimal criteria for defining multipotent mesenchymal stromal cells. The International Society for Cellular Therapy position statement. Cytotherapy 2006, 8: 315-7.

31. Carrancio S, Blanco B, Romo C, Muntion S, LópezHolgado N, Blanco JF, et al. Bone Marrow Mesenchymal Stem Cells for Improving Hematopoietic Function: An In Vitro and In Vivo Model. Part 2: Effect on Bone Marrow Microenvironment. PLoS ONE 2011; 6: e26241.

32. Majumdar MK, Thiede MA, Haynesworth SE, Bruder SP, Gerson SL. Human marrow-derived mesenchymal stem cells (MSCs) express hematopoietic cytokines and support long-term hematopoiesis when differentiated toward stromal and osteogenic lineages. J Hematother Stem Cell Res 2000; 9: 841-8.

33. López-Villar O, García J, Sánchez-Guijo F, Robledo C, Villaron E, Hernández-Campo $\mathrm{P}$, et al. Both expanded and uncultured mesenchymal stem cells from MDS patients are genomically abnormal, showing a specific genetic profile for the 5q- syndrome. Leukemia 2009; 23: 664-72.

34. Garayoa M, García JL, Santamaría C, García-Gómez A, Blanco JF, Pandiella A, et al. Mesenchymal stem 
cells from multiple myeloma patients display distinct genomic profile as compared with those from normal donors. Leukemia 2009; 23: 1515-27.

35. Zhao Z, Tang X, You Y, Li W, Liu F, Zou P. Assessment of bone marrow mesenchymal stem cell biological characteristics and support hemotopoiesis function in patients with chronic myeloid leukemia. Leuk Res 2006; 30: 993-1003.

36. Carrara RCV, Orellana MD, Fontes AM, Palma PVB, Kashima S, Mendes MR, et al. Mesenchymal stem cells from patients with chronic myeloid leukemia do not express BCR-ABL and have absence of chimerism after allogeneic bone marrow transplant. Braz J Med Biol Res 2007; 40: 57-67.

37. Fang B, Zheng C, Liao L, Han Q, Sun Z, Jiang X, et al. Identification of human chronic myelogenous leukemia progenitor cells with hemangioblastic characteristics. Blood 2005; 105: 2733-40.

38. Shirasaki R, Tashiro H, Oka Y, Matsuo T, Yamamoto $\mathrm{T}$, Sugao T, et al. Chronic Myelogenous Leukemia Cells Contribute to the Stromal Myofibroblasts in Leukemic NOD/SCID Mouse In Vivo. J Oncol 2012; 901783. doi: $10.1155 / 2012 / 901783$.

39. Vianello F, Villanova F, Tisato V, Lymperi S, Ho K-K, Gomes AR, et al. Bone marrow mesenchymal stromal cells non-selectively protect chronic myeloid leukemia cells from imatinib-induced apoptosis via the CXCR4/ CXCL12 axis. Haematologica 2010; 95: 1081-9.

40. Suzuki K, Sun R, Origuchi M, Kanehira MT, Akahata T, Itoh J, et al. Mesenchymal Stromal Cells Promote Tumor Growth through the Enhancement of Neovascularization. Mol Med 2011; 17: 579-87.

41. Corre J, Mahtouk K, Attal M, Gadelorge M, Huynh A, Fleury-Cappellesso S, et al. Bone marrow mesenchymal stem cells are abnormal in multiple myeloma. Leukemia 2007; 21: 1079-88.

42. Xishan Z, Guangyu A, Yuguang S, Hongmei Z. The research on the immuno-modulatory defect of mesenchymal stem cell from Chronic Myeloid Leukemia patients. J Exp Clin Cancer Res 2011; 30: 47.

43. Jin L, Tabe Y, Konoplev S, Xu Y, Leysath CE, Lu H, et al. CXCR4 up-regulation by imatinib induces chronic myelogenous leukemia (CML) cell migration to bone marrow stroma and promotes survival of quiescent CML cells. Mol Cancer Ther 2008; 7: 48-58.

44. Weisberg E, Azab AK, Manley PW, Kung AL, Christie $\mathrm{AL}$, Bronson R, et al. Inhibition of CXCR4 in CML cells disrupts their interaction with the bone marrow microenvironment and sensitizes them to nilotinib. Leukemia 2012; 26: 985-90. 